\title{
Elastodynamic Analysis of Antiplane Anisotropic INTERFACE CRACKS
}

\author{
By Chien-Ching $\mathrm{Ma}^{1}$ and Chih-Yaun Liao ${ }^{2}$
}

\begin{abstract}
The transient elastodynamic full-field response and the dynamic stress intensity factor of a semiinfinite interface crack lying between dissimilar anisotropic media subjected to a dynamic body force are investigated. At $t=0$, a concentrated antiplane dynamic point loading is suddenly applied at Medium 1 . The total wave field is due to the effect of this point loading and the scattering of the incident wave by the interface crack. We introduce a linear coordinate transformation that can transform the anisotropic interface crack problem to the isotropic case. The relationship between the problem of anisotropic material and the corresponding isotropic problem is established for shear stresses and displacement in a Cartesian coordinate system. Exact transient closed-form solutions for stresses and stress intensity factors are obtained. Numerical results for the time history of stresses and stress intensity factors during the transient process are discussed in detail.
\end{abstract}

\section{INTRODUCTION}

The asymptotic elastic fields of a semi-infinite crack lying along an interface between dissimilar isotropic materials subjected to static loading was first considered by Williams (1959) for a plane strain condition. He found that the stresses share the inverse square root singularity of the crack in a homogeneous material, and in addition, exhibit an oscillatory behavior as the interface crack tip is approached. Such an oscillatory behavior leads to the contradiction that the crack faces interpenetrate each other. Extensions to anisotropic elasticity for the near tip field have been made by Gotoh (1967), Bogy (1972), and Ting (1986, 1990). The exact full-field solutions of interface cracks for a plane problem in an anisotropic dissimilar media is obtained by Ma and Luo (1996). The antiplane problem of two dissimilar anisotropic wedges of arbitrary angles that are bonded together perfectly along a common edge has been considered by Ma and Hour (1989). A previous analysis of the dissimilar anisotropic antiplane wedge problem by Ma (1992) has shown that if an effective angle and effective material constant are introduced for the anisotropic case, then the order of singularity for the anisotropic wedge can be obtained from the result of the isotropic case. The results obtained in Ma (1992) have been extended by Ma (1996), where the correspondence relations of the fullfield solutions of stresses and displacement have been established for the anisotropic problem and for the isotropic problem in antiplane deformation.

In conventional studies of a semi-infinite crack in an unbounded homogeneous medium subjected to a spatially uniform dynamic traction distribution acting on crack faces, the complete solution is obtained by integral transform methods together with a direct application of the Wiener-Hopf technique and the Cagniard-de Hoop method of Laplace inversion. The evolution of the Wiener-Hopf technique and a number of extensions are described by Noble (1958). The application of the technique to transient problems in elastodynamics was pioneered by de Hoop (1958) in a study of several diffraction problems. If the loading is replaced by a nonuniform distribution having a characteristic length, then the Wiener-Hopf

${ }^{1}$ Prof., Dept. of Mech. Engrg., Nat. Taiwan Univ., Taipei, Taiwan 10617; corresponding author.

${ }^{2}$ Grad. Student, Dept. of Mech. Engrg., Nat. Taiwan Univ., Taipei, Taiwan 10617

Note. Associate Editor: John P. Dempsey. Discussion open until January 1,2000 . To extend the closing date one month, a written request must be filed with the ASCE Manager of Journals. The manuscript for this paper was submitted for review and possible publication on May 7 , 1998. This paper is part of the Journal of Engineering Mechanics, Vol. 125, No. 8, August, 1999. (C)ASCE, ISSN 0733-9399/99/0008-0891$0898 / \$ 8.00+\$ .50$ per page. Paper No. 18354 . method cannot be directly applied. Freund (1974) studied the problem of an elastic homogeneous solid containing a halfplane crack subjected to a pair of concentrated loadings on the crack faces at a finite distance from the crack tip. The transient response of a stationary semi-infinite isotropic interface crack subjected to dynamic body force was analyzed by Ma and Huang (1999), and the propagating case by Ing and Ma (1997) and Brock and Achenbach (1973). The elastodynamic analysis due to applying antiplane point loadings on the faces of an interface crack along dissimilar anisotropic materials was studied by Kuo and Cheng (1991).

In this study, the transient problem of a concentrated antiplane body force imposed suddenly near a semi-infinite anisotropic interface crack is investigated in detail. At time $t=$ 0 , an antiplane concentrated dynamic loading is applied at the interface anisotropic medium, as shown in Fig. 1. This problem can be treated as the superposition of two problems. The first problem is the disturbance by a concentrated force in the crack-free, unbounded anisotropic medium. The second problem considers the interface crack surfaces subjected to the negatives of the tractions induced by the first problem. To solve this problem, a linear coordinate transformation and a new fundamental solution are proposed in this study. The linear coordinate transformation can reduce the antiplane shear deformation in an anisotropic solid to the corresponding isotropic problem. The relationship has been noted by several researchers [e.g., Markenscoff and Ni (1984), Kuo and Cheng (1991), Wu and Chiu (1991), and Ma (1996)]. In particular, Markenscoff and $\mathrm{Ni}$ (1984) analyzed the elastodynamic responses of a homogeneous anisotropic solid. The proposed fundamental problem is one of applying an exponentially distributed traction on the interface crack faces in the Laplace transform domain, and the complete solution is determined by superposition of the fundamental solution. This alternative superposition scheme has also been used to successfully solve many transient problems for the interaction of incident cylindrical waves with cracks in a homogeneous medium; e.g., Tsai and $\mathrm{Ma}$ (1992) for a stationary crack, and Ma and Ing (1995) for a propagating crack. The transient full-field solutions of the anisotropic interface crack and the dynamic stress intensity factor are obtained and expressed in a closed form. Numerical results

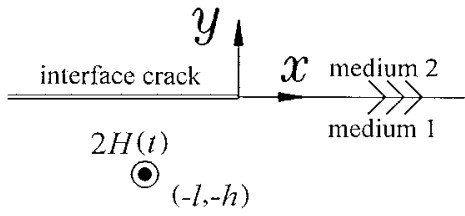

FIG. 1. Loading and Coordinate System of Interface Crack in Bimaterial Anisotropic Medium 
of stresses and dynamic stress intensity factors for the problem considered are evaluated and discussed in detail.

\section{LINEAR COORDINATE TRANSFORMATION}

For the absence of body force, the 2D antiplane wave motion of a homogeneous, anisotropic solid is governed by

$$
C_{55} \frac{\partial^{2} w^{a}}{\partial x^{2}}+2 C_{45} \frac{\partial^{2} w^{a}}{\partial x \partial y}+C_{44} \frac{\partial^{2} w^{a}}{\partial y^{2}}=\rho^{a} \frac{\partial^{2} w^{a}}{\partial t^{2}}
$$

where $w^{a}=$ only nonvanishing displacement component in the $z$-axis; $C_{i j}(i, j=4,5)=$ elastic moduli; and $\rho^{a}=$ mass density of the anisotropic material. The $x, y$-plane has been assumed to coincide with one of the planes of the material symmetry, such that inplane and antiplane deformation are uncoupled. The stress components are related to the displacement as follows:

$$
\begin{aligned}
& \tau_{y z}^{a}(x, y, t)=C_{44} \frac{\partial w^{a}(x, y, t)}{\partial y}+C_{45} \frac{\partial w^{a}(x, y, t)}{\partial x} \\
& \tau_{x z}^{a}(x, y, t)=C_{45} \frac{\partial w^{a}(x, y, t)}{\partial y}+C_{55} \frac{\partial w^{a}(x, y, t)}{\partial x}
\end{aligned}
$$

We introduce a special linear coordinate transformation

$$
\begin{gathered}
X=x-\frac{C_{45}}{C_{44}} y \\
Y=\frac{C_{e}}{C_{44}} y
\end{gathered}
$$

where $C_{e}=\sqrt{C_{44} C_{55}-C_{45}^{2}}$. Then the equation in (1) can be rewritten to the standard wave equation in the form

$$
C_{e} \frac{\partial^{2} w^{a}}{\partial X^{2}}+C_{e} \frac{\partial^{2} w^{a}}{\partial Y^{2}}=\frac{C_{44}}{C_{e}} \rho^{a} \frac{\partial^{2} w^{a}}{\partial t^{2}}
$$

Note that the mixed derivative disappears from the equation. The constitutive equations change to

$$
\tau_{y z}^{a}(x, y, t)=C_{44} \frac{\partial w^{a}(x, y, t)}{\partial y}+C_{45} \frac{\partial w^{a}(x, y, t)}{\partial x}=C_{e} \frac{\partial w^{a}(X, Y, t)}{\partial Y}
$$

$$
\begin{aligned}
& \tau_{x z}^{a}(x, y, t)=C_{45} \frac{\partial w^{a}(x, y, t)}{\partial y}+C_{55} \frac{\partial w^{a}(x, y, t)}{\partial x} \\
&=\left(\frac{C_{45}}{C_{44}}\right) C_{e} \frac{\partial w^{a}(X, Y, t)}{\partial Y}+\left(\frac{C_{e}}{C_{44}}\right) C_{e} \frac{\partial w^{a}(X, Y, t)}{\partial X}
\end{aligned}
$$

For a homogeneous isotropic material, the wave equation is

$$
\mu \frac{\partial^{2} w^{i}}{\partial X^{2}}+\mu \frac{\partial^{2} w^{i}}{\partial Y^{2}}=\rho^{i} \frac{\partial^{2} w^{i}}{\partial t^{2}}
$$

and the constitutive equations are

$$
\begin{aligned}
\tau_{Y Z}^{i}(X, Y, t) & =\mu \frac{\partial w^{i}(X, Y, t)}{\partial Y} \\
\tau_{X Z}^{i}(X, Y, t) & =\mu \frac{\partial w^{i}(X, Y, t)}{\partial X}
\end{aligned}
$$

If we compare (6)-(8) to (9)-(11), one can see that it is possible to obtain the solution for an anisotropic problem from a correspondent result for an isotropic problem by the linear transformation of the coordinate and replace the relevant material constant; i.e., by setting $C_{e}=\mu$ and $\rho^{i}=\left(C_{44} / C_{e}\right) \rho^{a}$. The relationship of displacement and shear stresses for an anisotropic solid and the correspondent isotropic solid are represented as

$$
\begin{gathered}
w^{a}(x, y, t)=w^{i}(X, Y, t) \\
\tau_{y z}^{a}(x, y, t)=\tau_{Y Z}^{i}(X, Y, t) \\
\tau_{X Z}^{a}(x, y, t)=\left(\frac{C_{e}}{C_{44}}\right) \tau_{X Z}^{i}(X, Y, t)+\left(\frac{C_{45}}{C_{44}}\right) \tau_{Y Z}^{i}(X, Y, t)
\end{gathered}
$$

in which $x-y$ and $X-Y=$ respective Cartesian coordinate systems in anisotropic and isotropic problems. There are several interesting features in the Cartesian coordinate transformation. For a straight line $\left(x_{1}, y_{0}\right),\left(x_{2}, y_{0}\right)$ parallel to the $x$ axis will still be a straight line $\left(X_{1}, Y_{0}\right),\left(X_{2}, Y_{0}\right)$ parallel to the $X$-axis and with the same length after transformation; i.e., $X_{2}$ $-X_{1}=x_{2}-x_{1}$.

\section{FUNDAMENTAL PROBLEM AND SOLUTIONS}

In this section, a useful fundamental problem is proposed and the associated fundamental solution is obtained by using the Wiener-Hopf technique. Materials 1 and 2 occupy the lower and upper half-spaces, respectively. The wave equations in two media are

$$
\begin{aligned}
& C_{55}^{(1)} \frac{\partial^{2} w^{(1)}}{\partial x^{2}}+2 C_{45}^{(1)} \frac{\partial^{2} w^{(1)}}{\partial x \partial y}+C_{44}^{(1)} \frac{\partial^{2} w^{(1)}}{\partial y^{2}}=\rho^{(1)} \frac{\partial^{2} w^{(1)}}{\partial t^{2}} \\
& C_{55}^{(2)} \frac{\partial^{2} w^{(2)}}{\partial x^{2}}+2 C_{45}^{(2)} \frac{\partial^{2} w^{(2)}}{\partial x \partial y}+C_{44}^{(2)} \frac{\partial^{2} w^{(2)}}{\partial y^{2}}=\rho^{(2)} \frac{\partial^{2} w^{(2)}}{\partial t^{2}}
\end{aligned}
$$

Introduce a linear coordinate transformation

$$
\begin{gathered}
X_{(i)}=x-\frac{C_{45}^{(i)}}{C_{44}^{(i)}} y, \quad i=1,2 \\
Y_{(i)}=\frac{C_{e}^{(i)}}{C_{44}^{(i)}} y
\end{gathered}
$$

where

$$
C_{e}^{(i)}=\sqrt{C_{44}^{(i)} C_{55}^{(i)}-\left(C_{45}^{(i)}\right)^{2}}
$$

The antiplane wave motions in the transformed coordinates are governed by the standard wave equations

$$
C_{e}^{(i)} \frac{\partial^{2} w^{(i)}}{\partial X_{(i)}^{2}}+C_{e}^{(i)} \frac{\partial^{2} w^{(i)}}{\partial Y_{(i)}^{2}}=\frac{C_{44}^{(i)}}{C_{e}^{(i)}} \rho^{(i)} \frac{\partial^{2} w^{(i)}}{\partial t^{2}}
$$

The solution for an exponentially distributed loading applied at the interface crack faces in the Laplace transform domain will be referred to as the fundamental solution. It is noted that along the interface crack faces and the interface we have $X_{(1)}=X_{(2)}=X$. Boundary conditions for the fundamental problem can then be described as follows:

$$
\bar{\tau}_{Y Z}^{(1)}\left(X, 0^{-}, p\right)=\bar{\tau}_{Y Z}^{(2)}\left(X, 0^{+}, p\right)=e^{p \eta X}, \quad-\infty<X<0
$$

where $p=$ Laplace transform parameter; and $\eta=$ a constant. The overbar symbol is used for denoting the transform on time $t$. The one-sided Laplace transform with respect to time and the two-sided Laplace transform with respect to $X$ are defined by

$$
\begin{gathered}
\bar{w}(X, Y, p)=\int_{0}^{\infty} w(X, Y, t) e^{-p t} d t \\
\tilde{w}(\xi, Y, p)=\int_{-\infty}^{\infty} \bar{w}(X, Y, p) e^{-p \xi X} d x
\end{gathered}
$$

The displacements and shear stresses must be continuous on the interface, which gives the following conditions on the interface:

$$
\bar{w}^{(1)}\left(X, 0^{-}, p\right)=\bar{w}^{(2)}\left(X, 0^{+}, p\right), \quad 0<X<-\infty
$$




$$
\bar{\tau}_{Y Z}^{(1)}\left(X, 0^{-}, p\right)=\bar{\tau}_{Y Z}^{(2)}\left(X, 0^{+}, p\right), \quad 0<X<-\infty
$$

The solutions of ordinary differential equations resulting from Laplace transformation of the partial differential equations in (19), which are bounded as $Y_{(1)} \rightarrow-\infty$ and $Y_{(2)} \rightarrow$ $+\infty$, are

$$
\begin{gathered}
\tilde{w}^{(1)}\left(\xi, Y_{(1)}, p\right)=\tilde{A}_{1}(\xi, p) e^{p \alpha_{1}(\xi) Y_{(1)}}, \quad Y_{(1)} \leq 0 \\
\tilde{w}^{(2)}\left(\xi, Y_{(2)}, p\right)=\tilde{A}_{2}(\xi, p) e^{-p \alpha_{2}(\xi) Y_{(2)}}, \quad Y_{(2)} \geq 0
\end{gathered}
$$

where

$$
\alpha_{i}(\xi)=\left(b_{i}^{2}-\xi^{2}\right)^{1 / 2}, \quad b_{i}=\frac{\sqrt{C_{44}^{(i)} \rho^{(i)}}}{C_{e}^{(i)}}, \quad i=1,2
$$

and $\tilde{A}_{1}$ and $\tilde{A}_{2}=$ unknown functions. The branch cuts of $\alpha_{i}$ are introduced to be from $\xi \rightarrow-\infty$ to $-b_{i}$, and from $\xi=b_{i}$ to $\xi \rightarrow+\infty$, so that $\operatorname{Re}\left(\alpha_{i}\right) \geq 0$ in the entire cut complex $\xi$-plane, where $\mathrm{Re}$ denotes the real part. Application of the Laplace transforms to boundary conditions (20)-(22) yields

$$
\begin{array}{ll}
\tilde{\tau}^{+}+\frac{1}{p(\eta-\xi)}=p C_{e}^{(1)} \alpha_{1}\left(\tilde{A}_{1}^{-}+\tilde{A}^{+}\right), & Y_{(1)}=0^{-} \\
\tilde{\tau}^{+}+\frac{1}{p(\eta-\xi)}=p C_{e}^{(2)} \alpha_{2}\left(\tilde{A}_{2}^{-}+\tilde{A}^{+}\right), & Y_{(2)}=0^{+}
\end{array}
$$

where $\tilde{\tau}^{+}$and $\tilde{A}^{+}=$unknown functions in the transform domain for the shear stress and displacement along the interface, respectively. Eliminating $\tilde{A}^{+}$from (25) and (26), and defining $\tilde{A}^{-}=\tilde{A}_{1}^{-}-\tilde{A}_{2}^{-}$, which corresponds to the transformed crackopening displacement, we have

$$
\tilde{A}^{-}=\frac{1}{p}\left(\frac{C_{e}^{(1)} \alpha_{1}(\xi)+C_{e}^{(2)} \alpha_{2}(\xi)}{C_{e}^{(1)} C_{e}^{(2)} \alpha_{1}(\xi) \alpha_{2}(\xi)}\right)\left[\tilde{\tau}^{+}+\frac{1}{p(\eta-\xi)}\right]
$$

Eq. (27) may be solved by factorization in much the same way that an equation of the standard Wiener-Hopf type is solved.

\section{Case 1: $b_{1}>b_{2}$}

At this point it is convenient to introduce a new function $S_{2}(\xi)$, by defining

$$
S_{2}(\xi)=\frac{C_{e}^{(1)} \alpha_{1}(\xi)+C_{e}^{(2)} \alpha_{2}(\xi)}{\left(C_{e}^{(1)}+C_{e}^{(2)}\right) \alpha_{1}(\xi)}
$$

The function $S_{2}(\xi)$ has the properties that $S_{2}(\xi) \rightarrow 1$ as $|\xi|$ $\rightarrow \infty$, and that $S_{2}(\xi)$ has neither zeros nor poles in the $\xi$-plane by cuts along $b_{2}<\xi<b_{1}$ and $-b_{1}<\xi<-b_{2}$. From the general product factorization method, $S_{2}(\xi)$ can be written as the product of two regular functions $S_{2}^{+}(\xi)$ and $S_{2}^{-}(\xi)$, where

$$
S_{2}^{ \pm}(\xi)=\exp \left\{-\frac{1}{\pi} \int_{b_{2}}^{b_{1}} \tan ^{-1}\left[\frac{C_{e}^{(2)} \sqrt{\delta^{2}-b_{2}^{2}}}{C_{e}^{(1)} \sqrt{b_{1}^{2}-\delta^{2}}}\right] \frac{1}{\delta \pm \xi} d \delta\right\}
$$

In view of the previous discussion, (27) may be rewritten as

$$
\begin{aligned}
& \frac{p \sqrt{b_{2}-\xi}}{S_{2}^{-}(\xi)} \tilde{A}^{-}-\frac{\kappa}{p(\eta-\xi)} \frac{S_{2}^{+}(\eta)}{\sqrt{b_{2}+\eta}} \\
& =\frac{\kappa S_{2}^{+}(\xi)}{\sqrt{b_{2}+\xi}} \tilde{\tau}^{+}+\frac{\kappa}{p(\eta-\xi)}\left[\frac{S_{2}^{+}(\xi)}{\sqrt{b_{2}+\xi}}-\frac{S_{2}^{+}(\eta)}{\sqrt{b_{2}+\eta}}\right]
\end{aligned}
$$

where

$$
\kappa=\frac{C_{e}^{(1)}+C_{e}^{(2)}}{C_{e}^{(1)} C_{e}^{(2)}}
$$

The left-hand side of this equation is regular for $\operatorname{Re}(\xi)<b_{2}$, whereas the right-hand side is regular for $\operatorname{Re}(\xi)>-b_{2}$. Applying the analytic continuation argument, therefore, each side of (29) represents one and the same entire function, e.g., $E(\xi)$. Consequently, from the Abel theorem, $E(\xi)$ vanishes completely, and we find

$$
\begin{gathered}
\tilde{A}_{1}(\xi, p)=\frac{S_{2}^{+}(\eta) \sqrt{b_{2}+\xi}}{p^{2} S_{2}^{+}(\xi) C_{e}^{(1)} \alpha_{1}(\xi) \sqrt{b_{2}+\eta}(\eta-\xi)} \\
\tilde{A}_{2}(\xi, p)=\frac{S_{2}^{+}(\eta)}{p^{2} S_{2}^{+}(\xi) C_{e}^{(2)} \sqrt{b_{2}-\xi \sqrt{b_{2}+\eta}}(\eta-\xi)} \\
\tilde{\tau}(\xi, p)=\frac{S_{2}^{+}(\eta) \sqrt{b_{2}+\xi}}{p S_{2}^{+}(\xi) \sqrt{b_{2}+\eta}(\eta-\xi)}
\end{gathered}
$$

In view of (23), (24), (30), and (31), we obtain the fundamental solution of shear stress in the Laplace transform domain as follows:

$$
\begin{aligned}
& \bar{\tau}_{Y Z}^{(1)}\left(X_{(1)}, Y_{(1)}, p\right)=\frac{1}{2 \pi i} \int \frac{S_{2}^{+}(\eta) \sqrt{b_{2}+\xi}}{S_{2}^{+}(\xi) \sqrt{b_{2}+\eta}(\eta-\xi)} e^{p\left[\alpha_{1}(\xi) Y_{(1)}+\xi X_{(1)}\right]} d \xi \\
& \bar{\tau}_{Y Z}^{(2)}\left(X_{(2)}, Y_{(2)}, p\right)=\frac{1}{2 \pi i} \int \frac{S_{2}^{+}(\eta) \sqrt{b_{2}+\xi}}{S_{2}^{+}(\xi) \sqrt{b_{2}+\eta}(\eta-\xi)} e^{-p\left[\alpha_{2}(\xi) Y_{(2)}-\xi X_{(2)}\right]} d \xi
\end{aligned}
$$

The corresponding result of the dynamic stress intensity factor expressed in the Laplace transform domain is

$$
\bar{K}_{I I I}(p, \eta)=\lim _{X \rightarrow 0} \sqrt{2 \pi X} \bar{\tau}_{Y Z}(X, 0, p)=\frac{\sqrt{2} S_{2}^{+}(\eta)}{\left(b_{2}+\eta\right)^{1 / 2} p^{1 / 2}}
$$

\section{Case 2: $b_{1}<b_{2}$}

Following the similar procedure as presented previously for case $1\left(b_{1}>b_{2}\right)$, the solution of shear stress is

$\bar{\tau}_{Y Z}^{(1)}\left(X_{(1)}, Y_{(1)}, p\right)=\frac{1}{2 \pi i} \int \frac{S_{1}^{+}(\eta) \sqrt{b_{1}+\xi}}{S_{1}^{+}(\xi) \sqrt{b_{1}+\eta}(\eta-\xi)} e^{p\left[\alpha_{1}(\xi) Y_{(1)}+\xi X_{(1)}\right]} d \xi$

$\bar{\tau}_{Y Z}^{(2)}\left(X_{(2)}, Y_{(2)}, p\right)=\frac{1}{2 \pi i} \int \frac{S_{1}^{+}(\eta) \sqrt{b_{1}+\xi}}{S_{1}^{+}(\xi) \sqrt{b_{1}+\eta}(\eta-\xi)} e^{-p\left[\alpha_{2}(\xi) Y_{(1)}-\xi X_{(1)}\right]} d \xi$

where

$$
S_{1}^{ \pm}(\xi)=\exp \left\{-\frac{1}{\pi} \int_{b_{1}}^{b_{2}} \tan ^{-1}\left[\frac{C_{e}^{(1)} \sqrt{\delta^{2}-b_{1}^{2}}}{C_{e}^{(2)} \sqrt{b_{2}^{2}-\delta^{2}}}\right] \frac{1}{\delta \pm \xi} d \delta\right\}
$$

The corresponding result of the stress intensity factor is

$$
\bar{K}(p, \eta)=\frac{-\sqrt{2} S_{1}^{+}(\eta)}{\left(b_{1}+\eta\right)^{1 / 2} p^{1 / 2}}
$$

\section{TRANSIENT SOLUTIONS IN TIME DOMAIN}

As shown in Fig. 1, a bimaterial medium is composed of two homogeneous, anisotropic, and linearly elastic solids. A semi-infinite interface crack lying between dissimilar anisotropic half-planes is initially stress-free and at rest. At time $t$ $=0$, an antiplane dynamic loading in the $z$-direction is applied at the lower half-plane $x=-l$ and $y=-h$ in material 1 . The time dependence of the concentrated loading is represented by the Heaviside step function $H(t)$. The dynamic stress intensity factor of the interface crack will be induced as the incident wave generated from the concentrated loading arrives at the crack tip.

2D antiplane wave motions of anisotropic elastic solids in two media are governed by

$$
\begin{aligned}
& C_{55}^{(1)} \frac{\partial^{2} w^{(1)}}{\partial x^{2}}+2 C_{45}^{(1)} \frac{\partial^{2} w^{(1)}}{\partial x \partial y}+C_{44}^{(1)} \frac{\partial^{2} w^{(1)}}{\partial y^{2}}+2 \delta(x+l) \delta(y+h) H(t) \\
& =\rho^{(1)} \frac{\partial^{2} w^{(1)}}{\partial t^{2}}
\end{aligned}
$$




$$
C_{55}^{(2)} \frac{\partial^{2} w^{(2)}}{\partial x^{2}}+2 C_{45}^{(2)} \frac{\partial^{2} w^{(2)}}{\partial x \partial y}+C_{44}^{(2)} \frac{\partial^{2} w^{(2)}}{\partial y^{2}}=\rho^{(2)} \frac{\partial^{2} w^{(2)}}{\partial t^{2}}
$$

The traction-free crack-face boundary conditions and the continuity of displacement and traction along the interface are represented as follows:

$$
\begin{gathered}
\tau_{y z}^{(1)}\left(x, 0^{-}, t\right)=\tau_{y z}^{(2)}\left(x, 0^{+}, t\right)=0, \quad x<0 \\
\tau_{y z}^{(1)}\left(x, 0^{-}, t\right)=\tau_{y z}^{(2)}\left(x, 0^{+}, t\right), \quad x>0 \\
w^{(1)}\left(x, 0^{-}, t\right)=w^{(2)}\left(x, 0^{+}, t\right), \quad x>0
\end{gathered}
$$

Introduce a linear coordinate transformation as indicated in (17) and (18), then the governing equations in (40) and (41) become

$$
\begin{gathered}
C_{e}^{(1)} \frac{\partial^{2} w^{(1)}}{\partial X_{(1)}^{2}}+C_{e}^{(1)} \frac{\partial^{2} w^{(1)}}{\partial Y_{(1)}^{2}}+2 \delta\left(X_{(1)}+l_{0}^{(1)}\right) \delta\left(Y_{(1)}+h_{0}^{(1)}\right) H(t) \\
=\frac{C_{44}^{(1)}}{C_{e}^{(1)}} \rho^{(1)} \frac{\partial^{2} w^{(1)}}{\partial t^{2}} \\
C_{e}^{(2)} \frac{\partial^{2} w^{(2)}}{\partial X_{(2)}^{2}}+C_{e}^{(2)} \frac{\partial^{2} w^{(2)}}{\partial Y_{(2)}^{2}}=\frac{C_{44}^{(2)}}{C_{e}^{(2)}} \rho^{(2)} \frac{\partial^{2} w^{(2)}}{\partial t^{2}}
\end{gathered}
$$

where

$$
l_{0}^{(1)}=l-\frac{C_{45}^{(1)}}{C_{44}^{(1)}} h, \quad h_{0}^{(1)}=\frac{C_{e}^{(1)}}{C_{44}^{(1)}} h
$$

The incident field of the wave generated by the concentrated loading expressed in the Laplace transform domain can be represented as follows:

$$
\begin{gathered}
\bar{\tau}_{Y Z}^{(1)}=-\frac{1}{2 \pi i} \operatorname{sgn}\left(Y_{(1)}+h_{0}^{(1)}\right) \int_{\Gamma_{\lambda}} e^{-p t_{i}} d \lambda \\
\bar{\tau}_{X Z}^{(1)}=\frac{1}{2 \pi i} \int_{\Gamma_{\lambda}} \frac{\lambda}{\alpha_{1}} e^{-p t_{i}} d \lambda
\end{gathered}
$$

where

$$
t_{i}=\alpha_{1}(\lambda)\left|Y_{(1)}+h_{0}^{(1)}\right|-\lambda\left(X_{(1)}+l_{0}^{(1)}\right)
$$

When we combine (33), (34), and (47), the stress fields $\tau_{Y Z}$ expressed in the Laplace transform domain can be represented as follows:

$$
\begin{aligned}
\bar{\tau}_{Y Z}^{(1)} & =-\frac{1}{4 \pi^{2}} \int_{\Gamma_{\eta}} \int_{\Gamma_{\xi}} G(\xi, \eta) \exp \left[p \alpha_{1}(\xi) Y_{(1)}+p \xi X_{(1)}\right] \\
\cdot & \exp \left[-p \alpha_{1}(\eta) h_{0}^{(1)}+p \eta l_{0}^{(1)}\right] d \xi d \eta \\
\bar{\tau}_{Y Z}^{(2)} & =-\frac{1}{4 \pi^{2}} \int_{\Gamma_{\eta}} \int_{\Gamma_{\xi}} G(\xi, \eta) \exp \left[-p \alpha_{2}(\xi) Y_{(2)}+p \xi X_{(2)}\right] \\
\cdot & \exp \left[-p \alpha_{1}(\eta) h_{0}^{(1)}+p \eta l_{0}^{(1)}\right] d \xi d \eta
\end{aligned}
$$

where

$$
\begin{gathered}
G(\xi, \eta)=\frac{f_{1 / 2}(\eta) S_{2}^{+}(\eta) \sqrt{b_{2}+\xi}}{S_{2}^{+}(\xi) \sqrt{b_{2}+\eta}(\eta-\xi)} \\
=\frac{2 C_{e}^{(2)} \sqrt{b_{2}+\xi} \sqrt{b_{2}-\eta}}{\left(C_{e}^{(1)}+C_{e}^{(2)}\right) \alpha_{1}(\eta) S_{2}^{+}(\xi) S_{2}^{-}(\eta)(\eta-\xi)}
\end{gathered}
$$

for Case $1\left(b_{1}>b_{2}\right)$ and

$$
\begin{aligned}
& G(\xi, \eta)=\frac{f_{1 / 2}(\eta) S_{1}^{+}(\eta) \sqrt{b_{1}+\xi}}{S_{1}^{+}(\xi) \sqrt{b_{1}+\eta}(\eta-\xi)} \\
& =\frac{2 C_{e}^{(2)} \sqrt{b_{1}+\xi}}{\left(C_{e}^{(1)}+C_{e}^{(2)}\right) S_{1}^{+}(\xi) S_{1}^{-}(\eta) \sqrt{b_{1}+\eta}(\eta-\xi)}
\end{aligned}
$$

for Case $2\left(b_{1}<b_{2}\right)$. In which

$$
f_{1 / 2}(\eta)=\frac{2 C_{e}^{(2)} \alpha_{2}(\eta)}{C_{e}^{(1)} \alpha_{1}(\eta)+C_{e}^{(2)} \alpha_{2}(\eta)}
$$

The total wave fields of this problem in the Laplace transform domain can be expressed as

$$
\begin{aligned}
\bar{\tau}_{Y Z}^{(1)} & =-\frac{1}{2 \pi i} \int_{\Gamma_{\lambda}}\left[\operatorname{sgn}\left(Y_{(1)}+h_{0}^{(1)}\right) \exp \left(-p t_{i}\right)-r_{1 / 2}(\lambda) \exp \left(-p t_{r}\right)\right] d \lambda \\
& -\frac{1}{4 \pi^{2}} \int_{\Gamma_{\eta}} \int_{\Gamma_{\xi}} G(\xi, \eta) \exp \left[p \alpha_{1}(\xi) Y_{(1)}+p \xi X_{(1)}\right] \\
\cdot & \exp \left[-p \alpha_{1}(\eta) h_{0}^{(1)}+p \eta l_{0}^{(1)}\right] d \xi d \eta \\
\bar{\tau}_{Y Z}^{(2)} & \left.=-\frac{1}{2 \pi i} \int_{\Gamma_{\lambda}} f_{1 / 2}(\lambda) \exp \left(-p t_{f}\right)\right] d \lambda-\frac{1}{4 \pi^{2}} \int_{\Gamma_{\eta}} \int_{\Gamma_{\xi}} G(\xi, \eta) \\
\cdot & \exp \left[-p \alpha_{2}(\xi) Y_{(2)}+p \xi X_{(2)}\right] \exp \left[-p \alpha_{1}(\eta) h_{0}^{(1)}+p \eta l_{0}^{(1)}\right] d \xi d \eta
\end{aligned}
$$

where

$$
\begin{gathered}
r_{1 / 2}(\lambda)=\frac{C_{e}^{(1)} \alpha_{1}(\lambda)-C_{e}^{(2)} \alpha_{2}(\lambda)}{C_{e}^{(1)} \alpha_{1}(\lambda)+C_{e}^{(2)} \alpha_{2}(\lambda)} \\
t_{r}=-\alpha_{1}(\lambda)\left(Y_{(1)}-h_{0}^{(1)}\right)-\lambda\left(X_{(1)}+l_{0}^{(1)}\right) \\
t_{f}=\alpha_{2}(\lambda) Y_{(2)}+\alpha_{1}(\lambda) h_{0}^{(1)}-\lambda\left(X_{(2)}+l_{0}^{(1)}\right)
\end{gathered}
$$

The first two terms with one integration in (54) represent the solution of incident and reflected waves from the interface in Material 1 for applying a dynamic point loading in a bimaterial medium without crack, and the first term in (55) represents the refracted wave in Material 2. The last term with two integrations in (54) and (55) represent the scattering waves generated due to the disturbance of the interface crack.

The first two terms in (54) and the first term in (55) represent the complete solution for incident, reflected, and refracted waves in Material 1 and Material 2, respectively, for applying a dynamic point loading in a bimaterial medium without crack. The transient solution of these waves in the time domain can be constructed by using the Cagniard-de Hoop method, the idea of the method is to deform the path of integration in the $\lambda$-plane in such a manner that the inverse Laplace transform of the integral along the new path of integration can be obtained by inspection. The new path casts the integrand in the desired form

$$
\begin{aligned}
& -\frac{1}{2 \pi i} \operatorname{sgn}\left(Y_{(1)}+h_{0}^{(1)}\right) \int_{\Gamma_{\lambda}} e^{-p t_{i}} d \lambda+\frac{1}{2 \pi i} \int_{\Gamma_{\lambda}} r_{1 / 2}(\lambda) e^{-p t_{r}} d \lambda \\
& =-\frac{1}{\pi} \operatorname{sgn}\left(Y_{(1)}+h_{0}^{(1)}\right) \int_{b_{1} R_{i}}^{\infty} \operatorname{Im}\left(\frac{\partial \lambda_{i}^{+}}{\partial t_{i}}\right) e^{-p t_{i}} d t_{i} \\
& +\frac{1}{\pi} \int_{b_{1} R_{r}}^{\infty} \operatorname{Im}\left[r_{1 / 2}\left(\lambda_{r}^{+}\right) \frac{\partial \lambda_{r}^{+}}{\partial t_{r}}\right] e^{-p t_{r}} d t_{r}
\end{aligned}
$$

where

$$
\begin{gathered}
\lambda_{i}^{+}=-\frac{t_{i}}{R_{i}} \frac{\left(X_{(1)}+l_{0}^{(1)}\right)}{R_{i}}+i \sqrt{\frac{t_{i}^{2}}{R_{i}^{2}}-b_{1}^{2}} \frac{\left(Y_{(1)}+h_{0}^{(1)}\right)}{R_{i}} \\
\lambda_{r}^{+}=-\frac{t_{r}}{R_{r}} \frac{\left(X_{(1)}+l_{0}^{(1)}\right)}{R_{r}}+i \sqrt{\frac{t_{r}^{2}}{R_{r}^{2}}-b_{1}^{2}} \frac{\left|Y_{(1)}+h_{0}^{(1)}\right|}{R_{r}} \\
R_{i}=\left[\left(X_{(1)}+l_{0}^{(1)}\right)^{2}+\left(Y_{(1)}+h_{0}^{(1)}\right)^{2}\right]^{1 / 2} \\
R_{r}=\left[\left(X_{(1)}+l_{0}^{(1)}\right)^{2}+\left(Y_{(1)}-h_{0}^{(1)}\right)^{2}\right]^{1 / 2}
\end{gathered}
$$


and

$$
-\frac{1}{2 \pi i} \int_{\Gamma_{\lambda}} f_{1 / 2}(\lambda) e^{-p t_{f}} d \lambda=\frac{1}{\pi} \int_{\Gamma_{f}}^{\infty} \operatorname{Im}\left[f_{1 / 2}\left(\lambda_{f}^{+}\right) \frac{\partial \lambda_{f}^{+}}{\partial t_{f}}\right] e^{-p t_{f}} d t_{f}
$$

where

$$
\frac{\partial \lambda_{f}^{+}}{\partial t_{f}}=\frac{-1}{\frac{\lambda_{f}^{+} Y_{(2)}}{\alpha_{2}\left(\lambda_{f}^{+}\right)}+\frac{\lambda_{f}^{+} h_{0}^{(1)}}{\alpha_{1}\left(\lambda_{f}^{+}\right)}+\left(X_{(2)}+l_{0}^{(1)}\right)}
$$

The transient solutions for these three waves expressed in time domain are as follows:

(1) Incident wave:

$$
-\frac{1}{\pi} \operatorname{sgn}\left(Y_{(1)}+h_{0}^{(1)}\right) \operatorname{Im}\left(\frac{\partial \lambda_{i}^{+}}{\partial t_{i}}\right) H\left(t_{i}-b_{1} R_{i}\right)
$$

(2) Reflected wave:

$$
\frac{1}{\pi} \operatorname{Im}\left[r_{1 / 2}\left(\lambda_{r}^{+}\right) \frac{\partial \lambda_{r}^{+}}{\partial t_{r}}\right] H\left(t_{i}-b_{1} R_{r}\right)
$$

(3) Refracted wave:

$$
-\frac{1}{\pi} \operatorname{Im}\left[f_{1 / 2}\left(\lambda_{f}^{+}\right) \frac{\partial \lambda_{f}^{+}}{\partial t_{f}}\right] H\left(t_{f}-T_{f}\right)
$$

Eqs. (54) and (55) constitute a double-inversion integral. Here the inverse transform is carried out using an extension of the Cagniard-de Hoop technique. This technique enables two successive inversions to be performed in one step. In this particular problem, the technique proposed by Harris (1980) is used to perform three successive inversions. Cagniard contours are introduced in both the $\eta$ - and $\xi$-plane for Material 1 by setting

$$
\begin{gathered}
t_{p}=\alpha_{1}(\eta) h_{0}^{(1)}-\eta l_{0}^{(1)} \\
t_{d_{1}}=-\alpha_{1}(\xi) Y_{(1)}-\xi X_{(1)}
\end{gathered}
$$

Eqs. (63) and (64) can be solved for $\eta$ and $\xi$ to yield

$$
\begin{gathered}
\eta^{ \pm}=-\frac{t_{p}}{R_{p}} \cos \Theta_{p} \pm i \sqrt{\frac{t_{p}^{2}}{R_{p}^{2}}-b_{1}^{2}} \sin \Theta_{p} \\
\xi^{ \pm}=-\frac{t_{d_{1}}}{R_{d_{1}}} \cos \Theta_{d_{1}} \pm i \sqrt{\frac{t_{d_{1}}^{2}}{R_{d_{1}}^{2}}-b_{1}^{2}} \sin \Theta_{d_{1}}
\end{gathered}
$$

where

$$
\begin{gathered}
\left.R_{p}=\left[\left(l_{0}^{(1)}\right)^{2}+h_{0}^{(1)}\right)^{2}\right]^{1 / 2}, \quad \Theta_{p}=\cos ^{-1}\left(l_{0}^{(1)} / R_{p}\right)=\sin ^{-1}\left(h_{0}^{(1)} / R_{p}\right) \\
R_{d_{\Gamma}}=\left(X_{(1)}^{2}+Y_{(1)}^{2}\right)^{1 / 2}, \quad \Theta_{d_{1}}=\cos ^{-1}\left(X_{(1)} / R_{d_{1}}\right)=-\sin ^{-1}\left(Y_{(1)} / R_{d_{1}}\right)
\end{gathered}
$$

in which $\left(R_{p}, \Theta_{p}\right)$ and $\left(R_{d_{1}}, \Theta_{d_{1}}\right)=$ polar coordinates of the source point and the field point in Material 1, respectively. For Material 2, we set the Cagniard contour in the $\xi$-plane

$$
t_{d_{2}}=\alpha_{2}(\xi) Y_{(2)}-\xi X_{(2)}
$$

Solve for $\xi$ to yield

$$
\xi^{ \pm}=-\frac{t_{d_{2}}}{R_{d_{2}}} \cos \Theta_{d_{2}} \pm i \sqrt{\frac{t_{d_{2}}^{2}}{R_{d_{2}}^{2}}-b_{2}^{2}} \sin \Theta_{d_{2}}
$$

where

$$
R_{d_{2}}=\left(X_{(2)}^{2}+Y_{(2)}^{2}\right)^{1 / 2}, \quad \Theta_{d_{2}}=\cos ^{-1}\left(X_{(2)} / R_{d_{2}}\right)=\sin ^{-1}\left(Y_{(2)} / R_{d_{2}}\right)
$$

in which $\left(R_{d_{2}}, \Theta_{d_{2}}\right)=$ polar coordinate of the field point in Material 2. The positive definitions of the angles $\Theta_{p}, \Theta_{d_{1}}$, and $\Theta_{d_{2}}$ are shown in Fig. 2.

Complete wavefronts for some interesting situations of Case

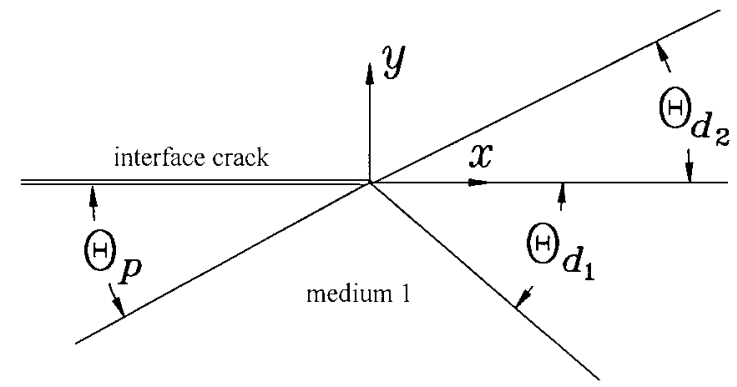

FIG. 2. Polar Coordinates for Source Point and Field Points in Materials 1 and 2

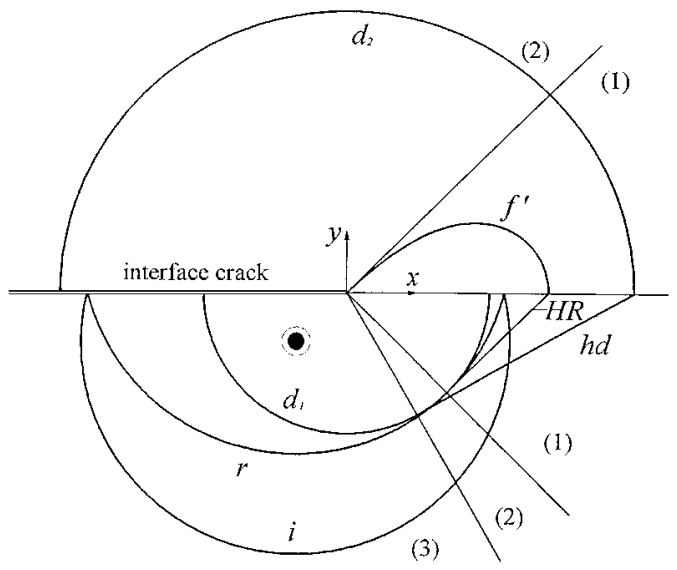

FIG. 3. Wavefronts of Incident, Reflected, Refracted, and Diffracted Waves with Loading Applied at $\Theta_{p}=\pi / 4, b_{2}=0.5 b_{1}$, and $t / b_{1} R_{p}=3$ for Case 1

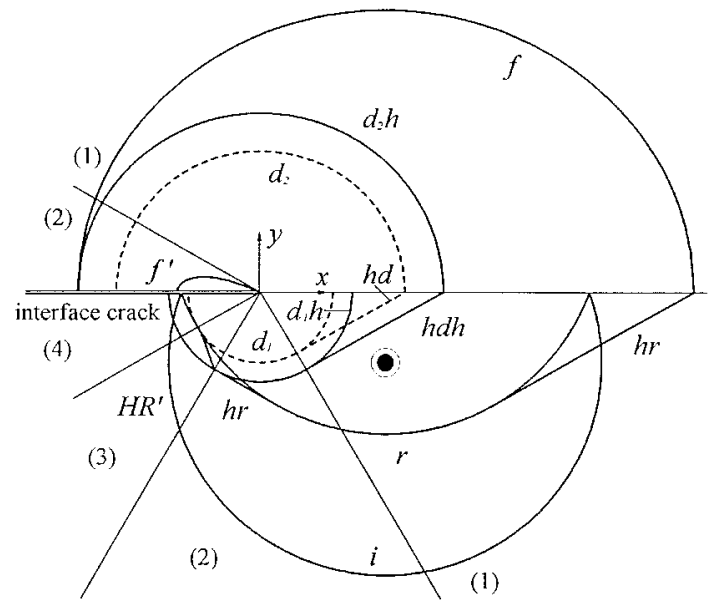

FIG. 4. Wavefronts of Incident, Reflected, Refracted, and Diffracted Waves with Loading Applied at $\Theta_{p}=5 \pi / 6, b_{2}=0.5 b_{1}$, and $t / b_{1} R_{p}=1.5$ for Case 1

1 are shown in Figs. 3 and 4, and for Case 2 in Figs. 5 and 6 , respectively. The waves shown in Figs. 3-6 are composed of incident waves, reflected waves, refracted waves, diffracted wave and head wave and are denoted by $i, r, f, d$, and $h$, respectively. The last term in (54) and (55) has a functional form possessing a pole at $\eta=\xi$, the contribution of the pole must be taken into account in the change of integral paths from $\eta$ to $t_{p}$ and $\xi$ to $t_{d_{1}}$. The requirement of $\operatorname{Re}(\eta)>\operatorname{Re}(\xi)$ dictates that the integral path $\Gamma_{\eta}$ is always located at the right-hand side of $\Gamma_{\xi}$. The wavefronts for Case $1\left(b_{1}>b_{2}\right)$ and $\Theta_{p}=\pi / 4$ is shown in Fig. 3 after the change of the $\Gamma_{\eta}$ and $\Gamma_{\xi}$ integrations into Cagniard contours; a pole at $\eta=\xi$ will be embraced for Regions (2) and (3), but no pole should be taken into account for Region (1) in Material 1. In Fig. 3 the refracted wave in Material 2 will be distorted and becomes $f^{\prime}$, and ap- 


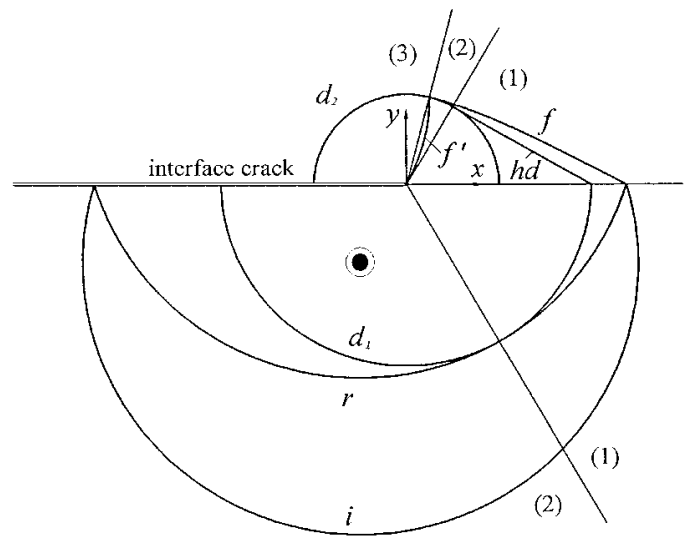

FIG. 5. Wavefronts of Incident, Reflected, Refracted, and Diffracted Waves with Loading Applied at $\Theta_{p}=\pi / 3, b_{2}=2 b_{1}$, and $t / b_{1} R_{p}=3$ for Case 2

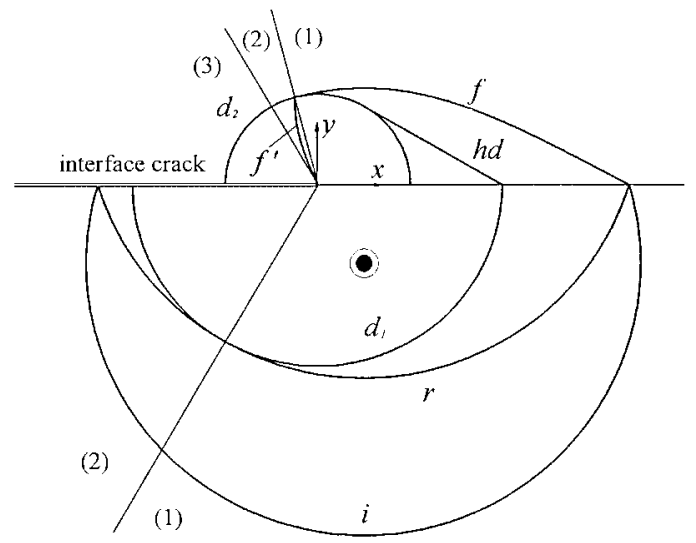

FIG. 6. Wavefronts of Incident, Reflected, Refracted, and Diffracted Waves with Loading Applied at $\Theta_{p}=2 \pi / 3, b_{2}=2 b_{1}$, and $t / b_{1} R_{p}=3$ for Case 2

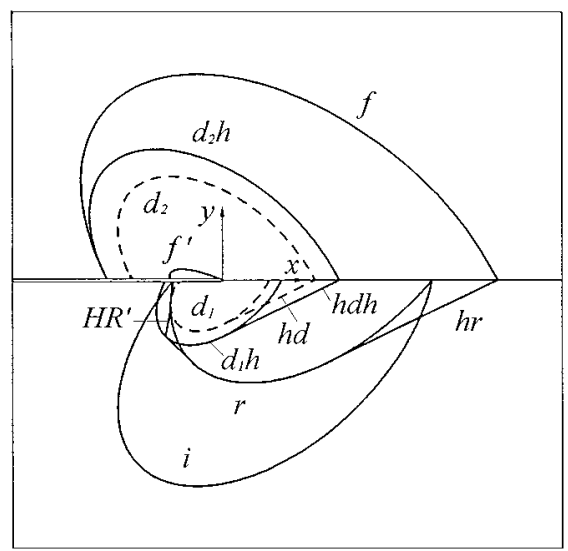

FIG. 7. Correspondent Wavefronts of Anisotropic Material for Special Material Combination $\left(C_{44}^{(1)}: C_{45}^{(1)}: C_{55}^{(1)}=2: 1: 2\right.$ and $\left.C_{44}^{(2)}: C_{45}^{(2)}: C_{55}^{(2)}=2:-1: 2\right)$ for Fig. 4

pears only at Region (1); i.e., $0<\Theta_{d_{2}}<\Theta_{p}$, there will be no refracted wave at Region (2). In Fig. 4 we have $\Theta_{p}=5 \pi / 6$, which corresponds to $\cos ^{-1}\left(-b_{2} / b_{1}\right)<\Theta_{p}<\pi$ and is the most complicated case. In this case the head wave $h r$ induced by the reflected $r$ wave will arrive at the crack tip before the incident $i$ wave, and additional diffracted waves $\left(d_{1} h, d_{2} h\right.$, and $h d$ waves) will be generated. The straight head wave will be deformed and terminated at the wavefront of the $d_{1}$ wave in Material 1 in Region (3) $\left(\cos ^{-1}\left(-b_{2} / b_{1}\right)<\Theta_{d_{1}}<\Theta_{p}\right)$. In Material 2 of Fig. 4, the normal refracted wave will occur at Region (1) $\left(0<\Theta_{d_{2}}<\Theta_{p}\right)$; however, the refracted wave is not complete and only exists before the extended $f^{\prime}$ wave from

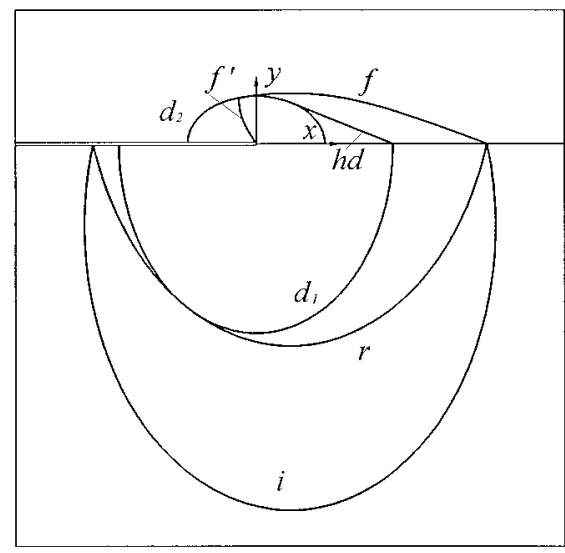

FIG. 8. Correspondent Wavefronts of Anisotropic Material for Special Material Combination $\left(C_{44}^{(1)}: C_{45}^{(1)}: C_{55}^{(1)}=2: 0: 1\right.$ and $\left.C_{44}^{(2)}: C_{45}^{(2)}: C_{55}^{(2)}=1: 0: 2\right)$ for Fig. 5

the crack tip arrives. There are only two situations for Case 2 $\left(b_{1}<b_{2}\right)$, Figs. 5 and 6 show the complete wavefronts for $\Theta_{p}$ $<\pi / 2$ and $\pi / 2<\Theta_{p}$, respectively. The correspondent wavefronts of anisotropic material for a special material combination for Figs. 4 and 5 are shown in Figs. 7 and 8, respectively.

The transient solutions of scattering waves in the time domain can be constructed by using the Cagniard-de Hoop technique. The solution of the diffracted wave for shear stress $\tau_{Y Z}$ is

$$
-\frac{1}{2 \pi^{2}} \int_{b_{1} R_{p}}^{t-b_{1} R_{d_{1}}} \operatorname{Re}\left[G\left(\xi^{+}, \eta^{+}\right) \frac{\partial \xi^{+}}{\partial t_{d_{1}}} \frac{\partial \eta^{+}}{\partial t_{p}}-G\left(\xi^{-}, \eta^{+}\right) \frac{\partial \xi^{-}}{\partial t_{d_{1}}} \frac{\partial \eta^{+}}{\partial t_{p}}\right] d t_{p}
$$

for the $d_{1}$ wave in Material 1 and

$$
-\frac{1}{2 \pi^{2}} \int_{b_{1} R_{p}}^{t-b_{2} R_{d_{2}}} \operatorname{Re}\left[G\left(\xi^{+}, \eta^{+}\right) \frac{\partial \xi^{+}}{\partial t_{d_{2}}} \frac{\partial \eta^{+}}{\partial t_{p}}-G\left(\xi^{-}, \eta^{+}\right) \frac{\partial \xi^{-}}{\partial t_{d_{2}}} \frac{\partial \eta^{+}}{\partial t_{p}}\right] d t_{p}
$$

for the $d_{2}$ wave in Material 2.

The dynamic stress intensity factors for isotropic and anisotropic material are defined as follows:

$$
\begin{gathered}
K^{i}(t)=\lim _{X \rightarrow 0^{+}} \sqrt{2 \pi X} \tau_{Y Z}(X, 0, t) \\
K^{a}(t)=\lim _{x \rightarrow 0^{+}} \sqrt{2 \pi x} \tau_{y z}(x, 0, t)
\end{gathered}
$$

As indicated in (4) and (13), we have $X=x$ and $\tau_{y z}^{a}=\tau_{Y Z}^{i}$ along the interface. Hence, the result of the stress intensity factor for the anisotropic problem is exactly the same as that obtained in the correspondent isotropic problem.

The dynamic stress intensity factors in the time domain for different situations can be expressed as follows.

\section{Case $1\left(b_{1}>b_{2}\right)$}

(1) $\cos ^{-1}\left(-b_{2} / b_{1}\right)>\Theta_{p}$ :

$$
K(t)=\sqrt{\frac{2}{\pi^{3}}} \int_{b_{1} R_{p}}^{t} \operatorname{Im}\left[\frac{f_{1 / 2}\left(\eta^{+}\right) S_{2}^{+}\left(\eta^{+}\right)}{\sqrt{b_{2}+\eta^{+}}} \frac{\partial \eta^{+}}{\partial \tau}\right] \frac{1}{\sqrt{t-\tau}} d \tau
$$

(2) $\pi>\Theta_{p}>\cos ^{-1}\left(-b_{2} / b_{1}\right)$ :

$$
K(t)=\sqrt{\frac{2}{\pi^{3}}} \int_{-b_{2} l_{0}^{(1)}+\left(b_{1}^{2}-b_{2}^{2}\right)^{1 / 2} h_{0}^{(1)}}^{t} \operatorname{Im}\left[\frac{f_{1 / 2}\left(\eta^{+}\right) S_{2}^{+}\left(\eta^{+}\right)}{\sqrt{b_{2}+\eta^{+}}} \frac{\partial \eta^{+}}{\partial \tau}\right] \frac{1}{\sqrt{t-\tau}} d \tau
$$

The result shown in (74) represents the situation that the head wave will arrive at the crack tip before the incident wave and 
the contribution from the head wave on the stress intensity factor should be taken into account.

\section{Case $2\left(b_{1}<b_{2}\right)$}

$$
K(t)=\sqrt{\frac{2}{\pi^{3}}} \int_{b_{1} R_{p}}^{t} \operatorname{Im}\left[\frac{f_{1 / 2}\left(\eta^{+}\right) S_{1}^{+}\left(\eta^{+}\right)}{\sqrt{b_{1}+\eta^{+}}} \frac{\partial \eta^{+}}{\partial \tau}\right] \frac{1}{\sqrt{t-\tau}} d \tau
$$

For the special case of homogeneous medium, i.e., $b_{1}=b_{2}$ and $C_{e}^{(1)}=C_{e}^{(2)}$, the solution of stress intensity factor can be reduced to that obtained by $\mathrm{Ma}$ and Chen (1993) as follows:

$$
K_{h}=\sqrt{\frac{2}{\pi R_{p}}} \cos \left(\Theta_{p} / 2\right)
$$

The interesting result represented in (76) is that the dynamic stress intensity factor for a semi-infinite crack in a homoge-

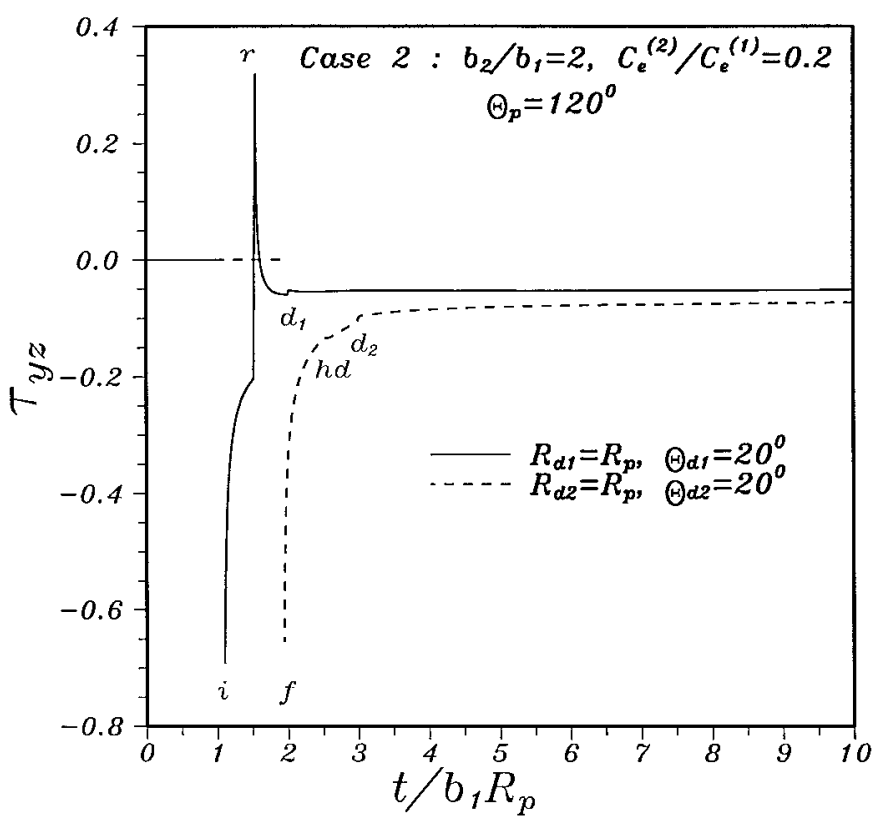

FIG. 9. Transient Stress $\tau_{y z}$ at Field Points $\boldsymbol{R}_{d_{1}}=\boldsymbol{R}_{p}, \Theta_{d_{1}}=20^{\circ}$ and $\boldsymbol{R}_{d_{2}}=\boldsymbol{R}_{p}, \Theta_{d_{2}}=20^{\circ}$ for Materials 1 and 2, Respectively, Subjected to Concentrated Force Applied at $\Theta_{p}=120^{\circ}$

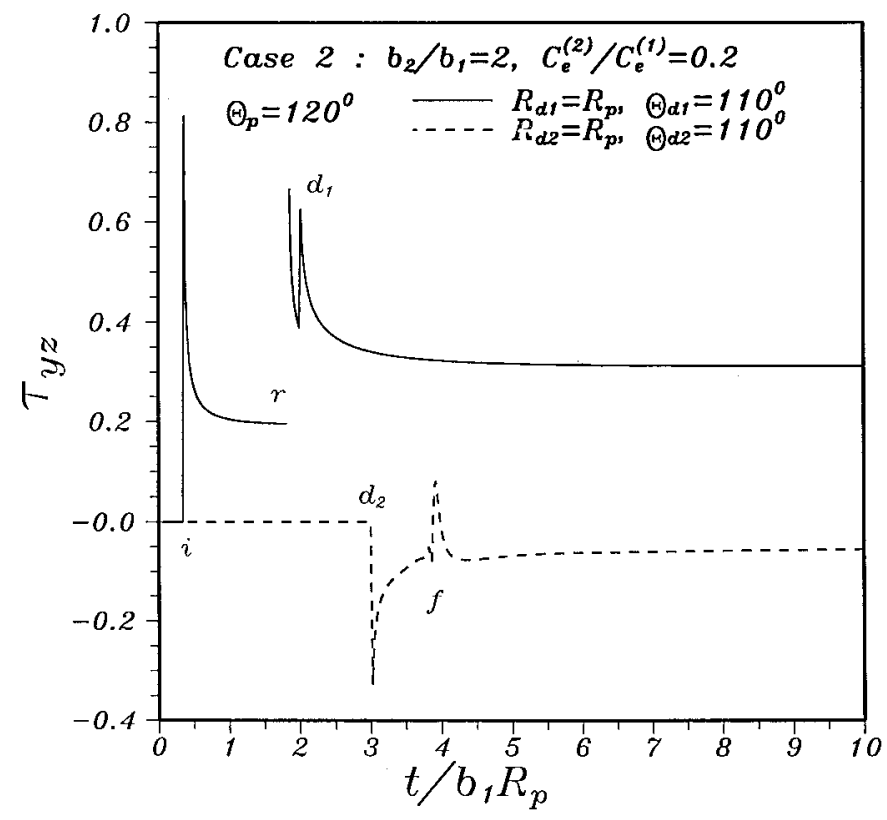

FIG. 10. Transient Stress $\tau_{y z}$ at Field Points $\boldsymbol{R}_{d_{1}}=\boldsymbol{R}_{p}, \Theta_{d_{1}}=110^{\circ}$ and $R_{d_{2}}=R_{p}, \Theta_{d_{2}}=110^{\circ}$ for Materials 1 and 2, Respectively, Subjected to Concentrated Force Applied at $\Theta_{p}=120^{\circ}$ neous medium jumps from zero to the corresponding static value after the incident shear wave generated from the loading point arrives at the crack tip.

\section{NUMERICAL RESULTS}

The transient response of stress for applying a concentrated force with Heaviside function dependence at the position $R_{p}$ and $\Theta_{p}=120^{\circ}$ is investigated first. The transient stresses evaluated at two field points at Materials 1 and 2 are shown in Figs. 9 and 10. Fig. 9 represents the transient response of the material point, $\Theta_{d_{1}}=20^{\circ}$ [Region (1)] at Material 1 and $R_{d_{2}}=$ $R_{p}, \Theta_{d_{2}}=20^{\circ}$ [Region (1)] at Material 2 for the case of $b_{2} / b_{1}$ $=2$ and $C_{e}^{(2)} / C_{0}^{(1)}=0.2$ (the corresponding complete wavefronts are shown in Fig. 6). Fig. 10 shows the transient response of stress at material point $R_{d_{1}}=R_{p}, \Theta_{d_{1}}=110^{\circ}$ [Region (1)] at Material 1 and $R_{d_{2}}=R_{p}, \Theta_{d_{2}}=110^{\circ}$ [Region (2)] at Material 2. After the last wave has passed the field point, the value of

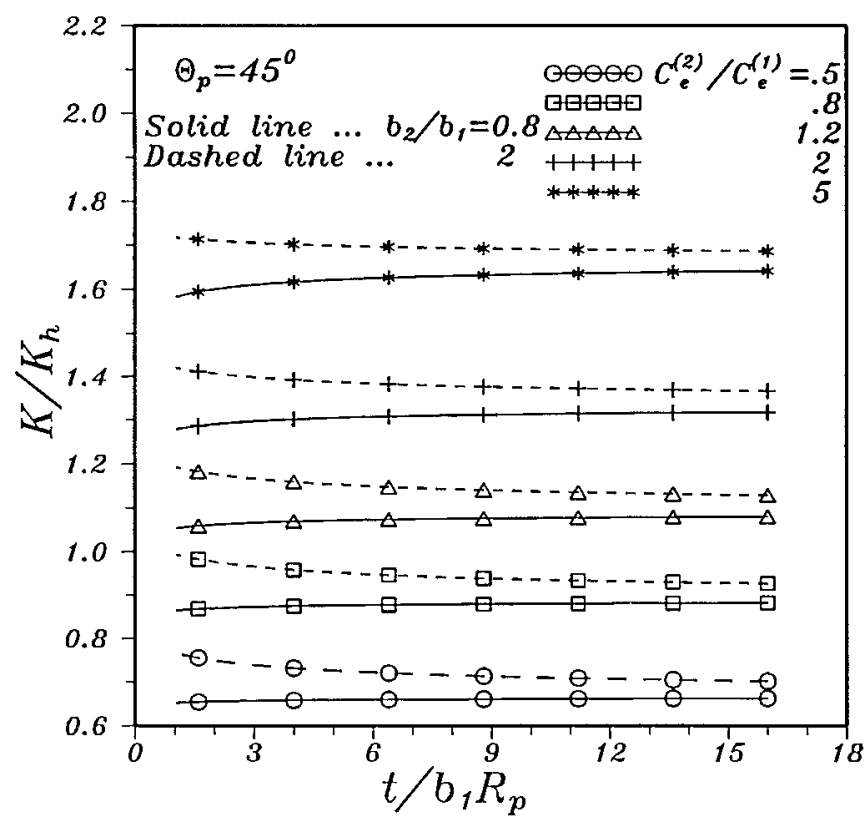

FIG. 11. Dynamic Stress Intensity Factors for $\Theta_{p}=45^{\circ}$ and Different Values of $C_{e}^{(2)} / C_{e}^{(1)}$

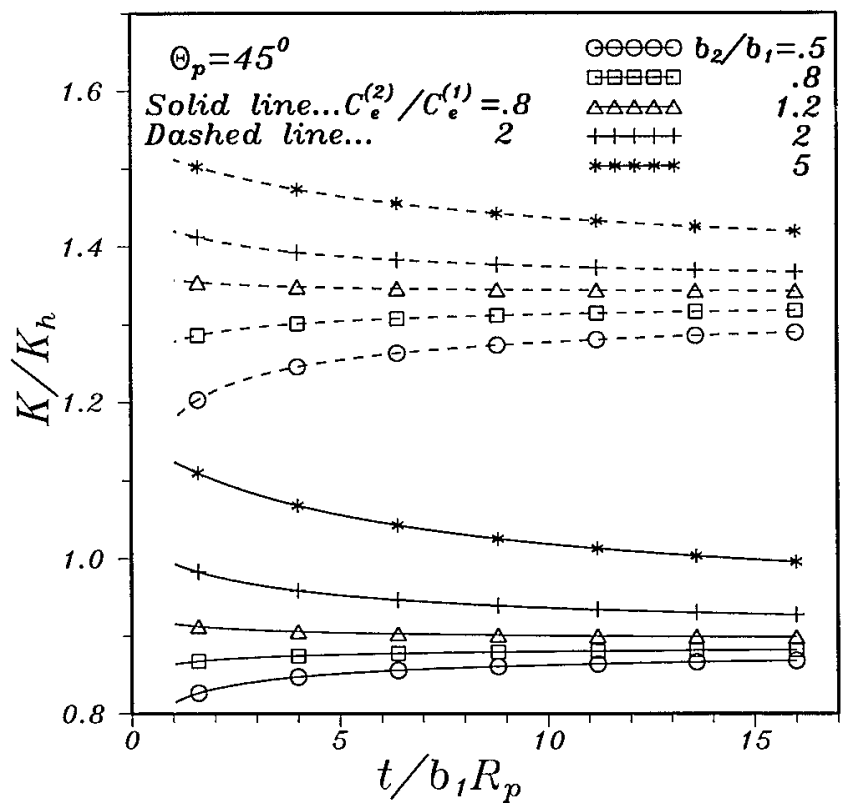

FIG. 12. Dynamic Stress Intensity Factors for $\Theta_{p}=45^{\circ}$ and Different Values of $b_{2} / b_{1}$ 
transient stresses will tend toward the corresponding static result.

The dynamic stress intensity factors for different situations are shown in Figs. 11 and 12. The dynamic stress intensity factor has been normalized by the corresponding result of the homogeneous case $K_{h}$. Figs. 11 and 12 show that the dynamic stress intensity factor will increase and approach a constant value for $b_{1}>b_{2}$, but decrease and approach a constant value for $b_{1}<b_{2}$.

\section{CONCLUSIONS}

For the mechanical behavior of many newly developed multiphase materials are mainly controlled by the response of the interface. Many researchers have investigated the field of dynamic debonding along a bimaterial interface. The transient problem of a semi-infinite interface crack in an anisotropic bimaterial is considered in this study. The exact transient solution has been obtained by integral transforms together with the direct application of the Wiener-Hopf technique and the Cagniard-de Hoop method. We introduce a linear coordinate transformation that transforms the anisotropic interface crack problem to an isotropic case. The geometric configuration in the isotropic case is the same as that in anisotropic material for the special linear coordinate transformation proposed in this study. The transient solutions for the isotropic problem are obtained by superposition of a proposed fundamental solution in the Laplace transform domain, and the solution for anisotropic problem can be constructed by using the relationship that has been established. The proposed fundamental solution is an exponentially distributed traction applied on the crack faces. Some interesting and important results are obtained in this study. We find that the dynamic stress intensity factor for the loading applied at the softer material $\left(C_{e}^{(2)}>C_{e}^{(1)}\right)$ will be larger than that applied at the harder material. For most cases of the loading applied at the softer material, the dynamic stress intensity factor will be larger than that for the homogeneous material. The results obtained in this investigation provide a great deal of information that is very important for the study of dynamic fractures.

\section{ACKNOWLEDGMENTS}

The writers gratefully acknowledge the financial support of this research by the National Science Council under Grant NSC 84-2212-E001062 .

\section{APPENDIX. REFERENCES}

Bogy, D. B. (1972). "The plane solution for anisotropic elastic wedges under normal and shear loading."' J. Appl. Mech., 39(4), 1103-1109.

Brock, L. M., and Achenbach, J. D. (1973). "Extension of an interface flaw under the influence of transient waves." Int. J. Solids and Struct., 9(1), 53-68.

de Hoop, A. T. (1958). "Representation theorems for the displacement in an elastic solid and their application to elastodynamic diffraction theory,"' PhD dissertation, Technische hoegschool, Delft, The Netherlands.

Freund, L. B. (1974). "The stress intensity factor due to normal impact loading of the faces of a crack." Int. J. Engrg. Sci., 12(2), 179-189.

Gotoh, H. (1967). "Some problems of bonded anisotropic plates with cracks along the bond." Int. J. Fracture, 3(4), 253-265.

Harris, J. G. (1980). "Diffraction by a crack of a cylindrical longitudinal pulse." J. Appl. Mathematics and Phys., 31, 367-383.

Ing, Y. S., and Ma, C. C. (1997). "Dynamic analysis of a propagating antiplane interface crack." J. Engrg. Mech., ASCE, 123(8), 783-791.

Kuo, M. K., and Cheng, S. H. (1991). "Elastodynamic responses due to anti-plane point impact loadings on the faces of an interface crack along dissimilar anisotropic materials.', Int. J. Solids and Struct., 28(6), 751-768.

Ma, C. C. (1992). "Antiplane problems of monoclinic material." $J$. Engrg. Mech., ASCE, 118(9), 1765-1782.

Ma, C. C. (1996). "Relationship of anisotropic and isotropic materials for antiplane problems." AIAA J., 34(11), 2453-2456.

Ma, C. C., and Chen, S. K. (1993). "Exact transient analysis of an antiplace semi-infinite crack subjected to dynamic body forces." Wave Motion, 17(2), 161-171.

Ma, C. C., and Hour, B. L. (1989). "Analysis of dissimilar anisotropic wedges subjected to antiplane shear deformation."' Int. J. Solids Struct., 25(11), 1295-1309.

Ma, C. C., and Huang, K. C. (1999). "Full field analysis of an anti-plane interface crack subjected to dynamic body forces." Int. J. Solids and Struct., 36(2), 285-309.

Ma, C. C., and Ing, Y. S. (1995). "Transient analysis of dynamic crack propagation with boundary effect."' J. Appl. Mech., 62(4), 1029-1038.

Ma, C. C., and Luo, J. J. (1996). "Plane solutions of interface cracks in anisotropic dissimilar media." J. Engrg. Mech., ASCE, 122(1), 30-38.

Markenscoff, X., and Ni, L. (1984). "The transient motion of a screw dislocation in an anisotropic medium." J. Elasticity, 14(1), 93-95.

Noble, B. (1958). The Wiener-Hopf technique. Pergamon, Tarrytown, New York.

Ting, T. C. T. (1986). "Explicit solution and invariance of the singularities at an interface crack in anisotropic composites." Int. J. Solids and Struct., 22(9), 965-983.

Ting, T. C. T. (1990). "Interface cracks in anisotropic bimaterials." $J$. Mech. Phys. Solids, 38(4), 505-313.

Tsai, C. H., and Ma, C. C. (1992). "Transient analysis of a semi-infinite crack subjected to dynamic concentrated forces.' J. Appl. Mech., 59(4), 804-811.

Williams, M. L. (1959). "The stress around a fault or crack in dissimilar media." Bull. Seismological Soc. of Am., 49(2), 199-204.

Wu, K. C., and Chiu, Y. T. (1991). "Antiplane shear interface cracks in anisotropic bimaterials.' J. Appl. Mech., 58(2), 399-403. 